\title{
The mitochondrial respiratory chain has a critical role in the antiviral process in Coxsackievirus B3-induced myocarditis
}

\author{
Linda Ebermann ${ }^{1,2}$, Sylwia Wika ${ }^{1}$, Inga Klumpe ${ }^{1,2}$, Elke Hammer $^{3}$, Karin Klingel $^{4}$, Dirk Lassner ${ }^{5}$, Uwe Völker ${ }^{3}$, \\ Ulrike Erben ${ }^{6}$, Heinz Zeichhardt ${ }^{7}$, Heinz-Peter Schultheiss ${ }^{1}$ and Andrea Dörner ${ }^{1}$
}

\begin{abstract}
Well-established differences in Coxsackievirus B3 (CVB3) elimination in resistant C57BL/6 and permissive A.SW/SnJ mice provide suitable models for studying the significance of the link between mitochondrial respiratory chain (RC), antioxidative stress components and mitochondrion-related apoptosis in the context of myocardial virus elimination. Distinct myocardial CVB3 titer in C57BL/6 $\left(2.5 \pm 1.4 \times 10^{4}\right.$ plaque-forming units (p.f.u.)/g tissue) and A.SW/SnJ mice $\left(1.4 \pm 0.8 \times 10^{7}\right.$ p.f.u./g) were associated with differences in the cardiac mitochondrial function 8 days post infection (p.i.). Infected C57BL/6 mouse hearts disclosed increased complex I (Cl) and CIII activity, but restricted CII and normal CIV activity of RC. Reduced expression of the antioxidative catalase was accompanied by elevated lipid peroxidation (LPO), indicating oxidative stress. Intrinsic apoptosis was activated demonstrated by elevated levels of Bax, Bcl-2, caspase 3 and DNA degradation. In contrast, all myocardial RC complex activities were restricted in CVB3-infected A.SW/SnJ mice.

The antioxidative system provided sufficient protection against oxidative stress shown by an elevated catalase expression and unaltered LPO. Bax and Bcl-2 levels were unchanged in CVB3-infected A.SW/SnJ mice, while caspase 3 was moderately increased but no DNA degradation was detectable. Correlation analyses including data from the two mouse strains revealed that reduced CVB3 titer correlated with increased $\mathrm{Cl}$ and $\mathrm{Clll}$ activity, oxidative stress as well as active apoptosis during acute myocarditis (MC). C57BL/6 mice completely eliminated CVB3 and inflammation and normalized all intracellular parameters, while A.SW/SnJ mice showed permanently restricted $\mathrm{Cl}$ activity in chronic MC 90 days p.i., at which time the replicating virus was no longer detectable but immunological processes were still active. Consequently, the regulation of energy metabolism appears crucial for an effective virus elimination and may be of prognostic and therapeutic significance for patients with virus-induced MC.

Laboratory Investigation (2012) 92, 125-134; doi:10.1038/labinvest.2011.145; published online 3 October 2011
\end{abstract}

KEYWORDS: apoptosis; Coxsackievirus B3; mitochondria; myocarditis; oxidative phosphorylation; oxidative stress

Coxsackievirus B3 (CVB3), a member of the enteroviral Picornaviridae, is one of the best-described infectious agents associated with myocarditis (MC). ${ }^{1}$ Interestingly, characteristics of the virus alone do not explain the vast difference in clinical outcomes of CVB3-induced MC. Acute inflammatory heart disease is either characterized by rapid virus elimination and recovery or ongoing chronic inflammation associated with virus persistence frequently resulting in dilated cardiomyopathy. ${ }^{2}$ Although it is widely acknowledged that the immunology of the host is crucial for the course of the disease, intracellular processes of the infected heart that determine the differences in the fate of the virus remain elusive.

Mitochondria, the center of cellular energy production, have been shown to be prominent targets of viral proteins, suggesting that they have a central role in the host's cellular defense system. ${ }^{3-5}$ The mitochondrial respiratory chain (RC), the antioxidative system and members of the $\mathrm{Bcl}-2$ family strongly influence the mitochondrial integrity and

\footnotetext{
${ }^{1}$ Department of Cardiology and Pneumonology, Charité-Campus Benjamin Franklin, Universitätsmedizin Berlin, Berlin, Germany; ${ }^{2}$ Institute of Biochemistry, Freie Universität Berlin, Berlin, Germany; ${ }^{3}$ Interfaculty Institute for Genetics and Functional Genomics, Ernst-Moritz-Arndt Universität, Greifswald, Germany; ${ }^{4}$ Department of Molecular Pathology, Universität Tübingen, Tübingen, Germany; ${ }^{5}$ Institute for Cardiac Diagnostic and Therapy (IKDT), Berlin, Germany; ${ }^{6}$ Department of Gastroenterology/Infectiology/Rheumatology, Charité-Universitätsmedizin Berlin, Campus Benjamin Franklin, Berlin, Germany and ${ }^{7}$ Department of Virology, Charité—Universitätsmedizin Berlin, Campus Benjamin Franklin, Berlin, Germany

Correspondence: Dr A Dörner, PhD, Department of Cardiology and Pneumonology, Charité-Universitätsmedizin Berlin, Campus Benjamin Franklin, Hindenburgdamm 30, 12200 Berlin, Germany.

E-mail: andrea.doerner@charite.de

Received 1 March 2011; revised 5 August 2011; accepted 16 August 2011
} 
consequently need to be examined to understand the link between mitochondrial function and CVB3 within the infected heart.

The RC, the final step of ATP production, transfers electrons from donor molecules via complexes I to IV (CI-CIV) to the acceptor molecule $\mathrm{O}_{2}$, generating the transmembrane electrochemical gradient used by the ATP synthase to synthesize ATP. Imbalance in this tightly coordinated system leads to misrouting of electrons and increased generation of reactive oxygen species (ROS) ${ }^{6,7}$ ROS are highly reactive and damages proteins, lipids and nucleic acids. Components of the antioxidative stress system such as mitochondrial superoxide dismutase (SOD2) and catalase activity neutralize these toxic radicals. Maladjustment in RC and disturbance of the antioxidative system result in mitochondrial instability and apoptosis. ${ }^{8}$ In those cases, members of the Bcl-2 family specifically modulate mitochondrial processes initiating programmed cell death. ${ }^{9}$ While Bcl-2 suppresses apoptosis, Bax increases the permeability of the inner mitochondrial membrane, induces the release of proapoptotic factors that activate the caspase cascade, initiating cellular degradation.

We hypothesize here that the regulation of mitochondrial function has a significant impact on the myocardial virus load in CVB3-induced MC. To test this hypothesis, we used two mouse strains that have been well described for their different capacity to eliminate CVB3 from the heart. While CVB3-infected C57BL/6 mice rapidly eliminate the virus and recover from MC, A.SW/SnJ mice develop chronic MC based on virus persistence. ${ }^{10,11}$ These mouse models closely reflect the etiopathologies in human CVB3-induced MC.

\section{MATERIALS AND METHODS Model of Murine MC}

All animal studies were performed according to national guidelines and were approved by the institutional animal care committee. The investigation conforms to the 'Guide for the Care and Use of Laboratory Animals' stated by the US National Institutes of Health. Transfection-derived CVB3 (Nancy strain, ATCC VR-30) was propagated on HeLa cells, isolated and stored at $-80^{\circ} \mathrm{C}$ until use. Sixteen 6-week-old male C57BL/6 mice and 16 age-matched mice of the A.SW/ SnJ background were intraperitoneally inoculated with $5 \times 10^{5}$ plaque-forming units (p.f.u.) of CVB3 in $200 \mu \mathrm{l}$ of phosphate-buffered saline (PBS). A subgroup of age-matched C57BL/6 and A.SW/SnJ mice sham-infected with $200 \mu \mathrm{l}$ PBS served as controls. Eight infected and uninfected mice of each strain were killed during the acute phase of MC at day 8. An additional set of animals was analyzed in the chronic phase 90 days post infection (p.i.).

\section{Assessment of Heart Inflammation and Viral Load}

The grade of myocardial inflammation was histologically determined by evaluating hematoxylin/eosin-stained paraffin sections as described previously by three independent investigators. ${ }^{12}$ The severity of heart inflammation was graded 0 for no, 1 for low, 2 for medium and 3 for extensive immune cell infiltrations. Myocardial and cellular CVB3 titers were determined by plaque-forming assay as previously described. ${ }^{12}$

\section{RNA Preparation and Real-Time PCR}

Total RNA was isolated from myocardium using TRIzol reagent (Invitrogen, Karlsruhe, Germany), and cDNA was generated using standard protocols. Quantitative real-time PCR was performed to quantify mRNA expression of tumor necrosis factor $\alpha(\mathrm{TNF} \alpha)$, interleukin $1 \beta$ (IL-1 $\beta)$ and transforming growth factor $\beta$-1 (TGF $\beta)$ using the Mastercycler realplex system (Eppendorf, Hamburg, Germany) and the respective TaqMan Gene Expression Assays from Applied Biosystems (Darmstadt, Germany). Gene expression was normalized in relation to the endogenous housekeeping gene hypoxanthine phosphoribosyltransferase 1 (HPRT1).

\section{Mitochondrion Isolation}

Heart tissue was disrupted with scissors in 10 volumes of isolation buffer containing $300 \mathrm{mM}$ sucrose, $5 \mathrm{mM}$ 3-( $N$-morpholino)propanesulfonic acid, $1 \mathrm{mM}$ glycol-bis (2-aminoethylether)- $N, N, N^{\prime}, N^{\prime}$-tetraacetic acid, $1 \mathrm{mg} / \mathrm{ml}$ bovine serum albumin, protease inhibitor mix diluted 1:100 (Sigma-Aldrich, Steinheim, Germany) pH 7.4. Tissue was homogenized in a glass potter and subsequently centrifuged at $1000 \mathrm{~g}$ for $10 \mathrm{~min}$ at $4{ }^{\circ} \mathrm{C}$. The supernatant was centrifuged at $12000 \mathrm{~g}$ for $30 \mathrm{~min}$ for mitochondrion preparation. The mitochondrial pellet was washed in $200 \mu$ l isolation buffer and again centrifuged at $12000 \mathrm{~g}$ for $30 \mathrm{~min}$. The mitochondria were resuspended in $50 \mu \mathrm{l}$ isolation buffer. The supernatant was centrifuged for the preparation of cytosolic proteins at $16000 \mathrm{~g}$ for $1 \mathrm{~h}$. Protein concentration was determined by the bicinchoninic acid test (Pierce, Bonn, Germany).

\section{Determination of RC Complex Activities}

CI activity was measured spectrophotometrically by the oxidation of NADH with decylubiquinone at $340 \mathrm{~nm}$, CII activity by the oxidation of succinate with ubiquinone at $600 \mathrm{~nm}$, CIII by the reduction of cytochrome c with decylubiquinol at $550 \mathrm{~nm}$ and CIV by the oxidation of reduced cytochrome $\mathrm{c}$ at $550 \mathrm{~nm}$, as described in Jarreta et al. ${ }^{13}$

\section{Mass-Spectrometry Analyses}

Mitochondrial proteins of individual mice ( $n=4$ per group) were digested by trypsin and the resulting peptide extracts were desalted on reverse phase material. ${ }^{14}$ LC-ESI-MS/MS analysis was performed on a LTQ-Orbitrap mass spectrometer (Thermo Scientific, Bremen, Germany). Proteins displaying different levels in the two mouse strains were obtained by label-free quantification analysis performed with Rosetta Elucidator version 3.3.0.1.39 (http://www. rosettabio.com/products/elucidator; Rosetta Biosoftware, MA, USA). 


\section{Western Blot Analysis}

Mitochondrial $(3 \mu \mathrm{g})$ and cytosolic $(30 \mu \mathrm{g})$ proteins were separated by electrophoresis on $4-12 \%$ polyacrylamide gels and blotted onto a polyvinylidenefluoride membrane. Western blots were performed using a standard protocol with specific primary antibodies against SOD2 (Abcam, Cambridge, UK), catalase (Acris, Herford, Germany), Bax, Bcl-2 (New England Biolabs, Frankfurt, Germany) and caspase 3 (BD Pharmingen, Heidelberg, Germany), and with HRPconjugated swine anti-rabbit as secondary antibody (1:10 000, Dako, Glostrup, Denmark). Specific signals were normalized against total loaded protein visualized by Coomassie blue staining.

\section{Detection of LPO}

Myocardial lipid peroxidation (LPO), an indicator for oxidative stress, was measured using the commercially available kit LPO 586 (Oxis International, Portland, OR, USA), which spectroscopically determined free malondialdehyde and 4-hydroxyalkenals at $586 \mathrm{~nm}$ absorbance. Tissue homogenate was generated in 10 volume ice-cold PBS according to the manufacturer's protocol.

\section{TUNEL Staining}

A terminal deoxynucleotidyl-transferase-mediated dUTP nick end-labeling (TUNEL) assay was used to identify double-stranded DNA fragmentation and the characteristics of DNA degradation by apoptosis. TUNEL staining was purchased by Roche Diagnostics Deutschland $\mathrm{GmbH}$ (Mannheim, Germany). Protease K was used for cell permeabilization. Alkaline phosphatase-coupled second antibody and Fast Red were used as a detection system according to the manufacturer's protocol. Positive control of the TUNEL assay was confirmed by treating the heart sections with DNase. Negative control of the TUNEL assay was confirmed by staining the heart tissue in the same manner without primary antibody.

\section{Statistical Evaluation}

Two groups were compared using the Student's $t$-test or Mann-Whitney $U$-test. Multiple groups were compared using the one-way ANOVA with the Newmann-Keuls test as post test. Differences were considered to be significant when $P<0.05$. Values are shown as the mean \pm s.e.m. The relationship between two parameters was determined by the 'Pearson correlation'.

\section{RESULTS}

\section{Myocardial Inflammation and CVB3 Load}

To validate the induction of MC by CVB3 application in the two mouse models, we histologically assessed the myocardial pathology. CVB3 infection led to a $100 \%$ incidence of inflammation in both C57BL/6 and A.SW/SnJ mouse hearts with an equal degree of myocardial infiltration 8 days p.i. (Figures $1 \mathrm{a}$ and $\mathrm{b}$ ). However, the quality of the immune response differed in the strains as seen by diverse cytokine transcription patterns. Although TNF $\alpha$ mRNA was equally transcribed in the two mouse strains (Figure 1c), IL-1 $\beta$ (Figure 1d) and TGF $\beta$ (Figure 1e) revealed a lower transcription level in A.SW/SnJ than in C57BL/6 mice. TGF $\beta$ did not change yet in infected A.SW/SnJ mice at the analyzed time point. Simultaneously, the myocardial titer of replicating CVB3 determined by plaque-forming assay was significantly higher in CVB3-permissive A.SW/SnJ than in resistant C57BL/6 mice (Figure 1f).

\section{Activities of Electron-Transporting RC Complexes}

To analyze how cardiac mitochondrial $\mathrm{RC}$ is regulated in CVB3-induced MC, activities of the electron-transporting RC CI-CIV were determined in mitochondria isolated from infected and uninfected C57BL/6 and A.SW/SnJ mice. Activities were similar in uninfected C57BL/6 and A.SW/SnJ control hearts. However, they displayed profoundly different patterns in the two mouse strains after CVB3 infection (Figure 2). CI and CIII activity were remarkably elevated in infected C57BL/6 mouse hearts by $21.7 \pm 6.1 \%(P<0.05)$ and $44.0 \pm 10.3 \%(P<0.05)$, respectively, while CII activity was moderately restricted by $12.9 \pm 4.8 \%(P<0.05)$ and CIV showed normal functionality (Figure $2 \mathrm{a}$ ). In contrast, CVB3infected A.SW/SnJ hearts revealed a coordinate decrease in all RC complex activities of $16 \%$ on average (Figure $2 \mathrm{~b}$ ) compared with uninfected controls.

To elucidate whether changes in RC complex activities were related to an altered expression level of RC complex subunits, mass-spectrometric analyses were performed on mitochondrial protein from infected C57BL/6 and A.SW/SnJ hearts. Subunits whose expression differed are listed in Table 1. Consistent with the observed increase in CI activity in infected C57BL/6 hearts, 11 of $45 \mathrm{CI}$ subunits showed a higher protein content in C57BL/6 than in A.SW/SnJ mitochondria. The amount of the CII subunit SDHC was lower in the infected C57BL/6 mice. In all, 3 of 11 CIII subunits, UQCRS1, CYC1 and UQCR10, revealed a lower protein amount in infected C57BL/6 than in A.SW/SnJ hearts. In total, 2 of $13 \mathrm{CIV}$ subunits, COX7A2 L and COX7A1, were remarkably lower in infected C57BL/6 than in A.SW/SnJ hearts, although CIV activity showed normal function in infected C57BL/6 hearts.

Hence, the patterns in expression and function of the mitochondrial electron-transporting RC complexes are strain-specifically regulated in virus-induced MC.

\section{Myocardial Expression of Antioxidative Stress Enzymes and LPO}

An imbalance in RC complex activities is frequently linked to increased ROS generation that needs to be neutralized by the antioxidative stress system to avoid oxidative damage. As markers for an active antioxidative stress system, we analyzed the mitochondrial SOD2 and the cytosolic catalase protein amount as well as LPO as an indicator for oxidative damage. 

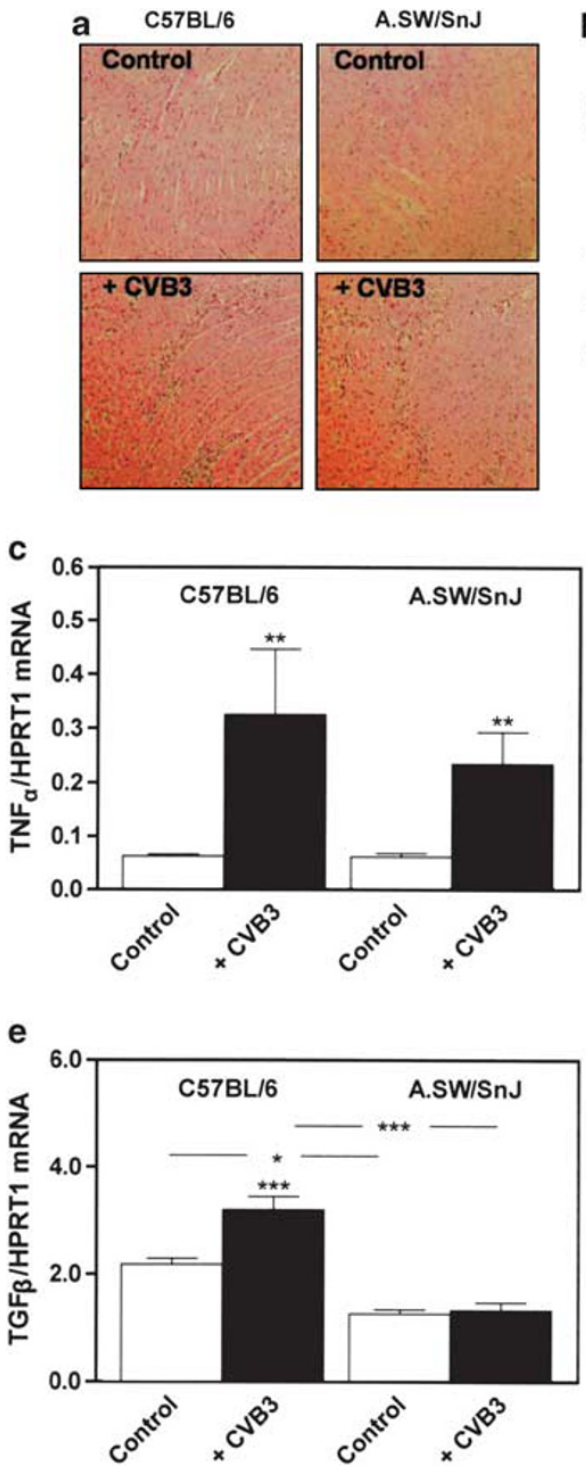
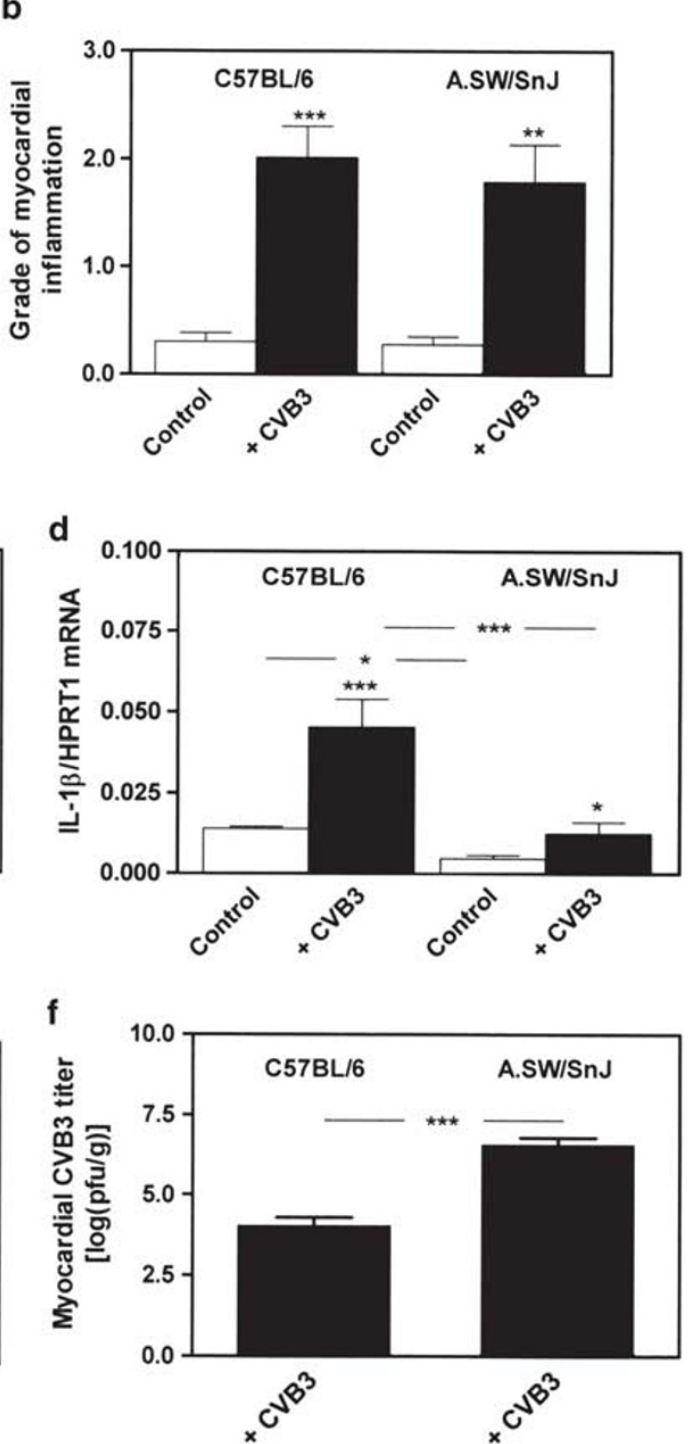

Figure 1 Hematoxylin/eosin-stained heart sections from uninfected (control) and infected (+ CVB3) C57BL/6 and A.SW/SnJ mice were analyzed for MC at day 8 post infection (p.i.) (a). The severity of MC was graded 0 for no, 1 for low, 2 for medium and 3 for high infiltration with immunocompetent cells. The averages are shown in (b). Open bars show uninfected age-matched controls, filled bars stand for infected animals. The myocardial transcription pattern of energy metabolism-relevant cytokines $\operatorname{TNF} \alpha(\mathbf{c}), \mathrm{IL}-1 \beta$ (d) and $\operatorname{TGF} \beta$ (e) was determined by quantitative PCR in the two mouse strains. Gene expression was normalized in relation to the endogenous housekeeping gene hypoxanthine phosphoribosyltransferase 1 (HPRT1). Myocardial CVB3 titer was analyzed by plaque assay from infected C57BL/ 6 and A.SW/SnJ mice 8 days p.i. (f). ${ }^{\star} P<0.05 ;{ }^{* * P}<0.01 ;{ }^{* * *} P<0.001$.

During infection, the amount of mitochondrial SOD2 increased by $29.0 \pm 7.9 \%(P<0.01)$, while cytosolic catalase expression decreased to $41.2 \pm 6.5 \%(P<0.01)$ in infected C57BL/6 hearts (Figures 3a and b) compared with controls. This was accompanied by increased oxidative damage demonstrated by an elevation of LPO of $38.0 \pm 14.2 \%$ $(P<0.05)$ (Figure $3 \mathrm{c}$ ).

In contrast, in comparison with uninfected controls CVB3infected A.SW/SnJ hearts doubled the catalase content $(206.7 \pm 18.9 \%$; $P<0.01)$ but reduced their SOD2 expression by $26.3 \pm 2.9 \%(P<0.01)$ (Figures $3 \mathrm{~d}$ and $\mathrm{e}$ ), which was still as high as that seen in infected C57BL/6 mouse hearts. The LPO level remained unaffected in the infected A.SW/SnJ hearts (Figure 3f). Hence, CVB3-infected C57BL/6 hearts were subjected to oxidative stress, whereas infected A.SW/SnJ hearts were protected from LPO by sufficiently expressing antioxidative enzymes.

\section{Expression of Mitochondrion-Related Apoptosis Markers in the Acute Phase of MC}

To further clarify whether deregulation in RC activities and oxidative stress were associated with mitochondrion-related apoptosis, pro- and antiapoptotic markers were examined during the acute phase of MC. In comparison with uninfected controls, infected C57BL/6 mice displayed significantly elevated myocardial Bax $(150.6 \pm 13.2 \% ; P<0.01)$ and $\mathrm{Bcl}-2$ 
a

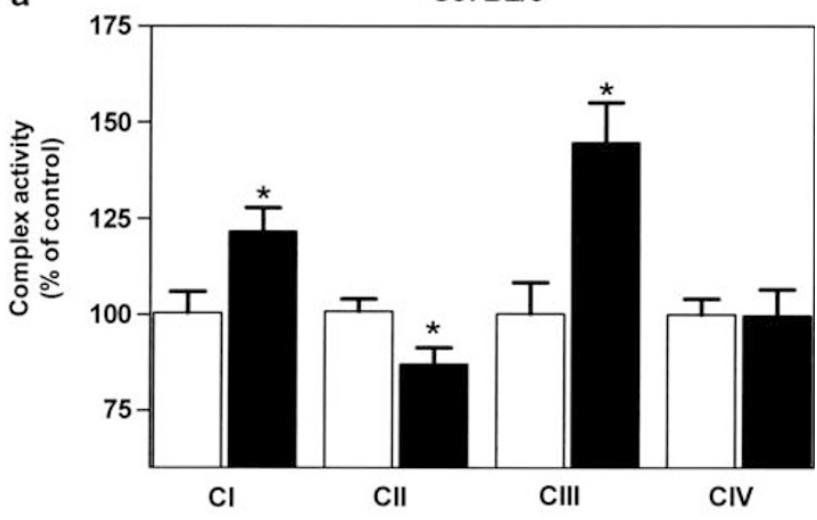

b

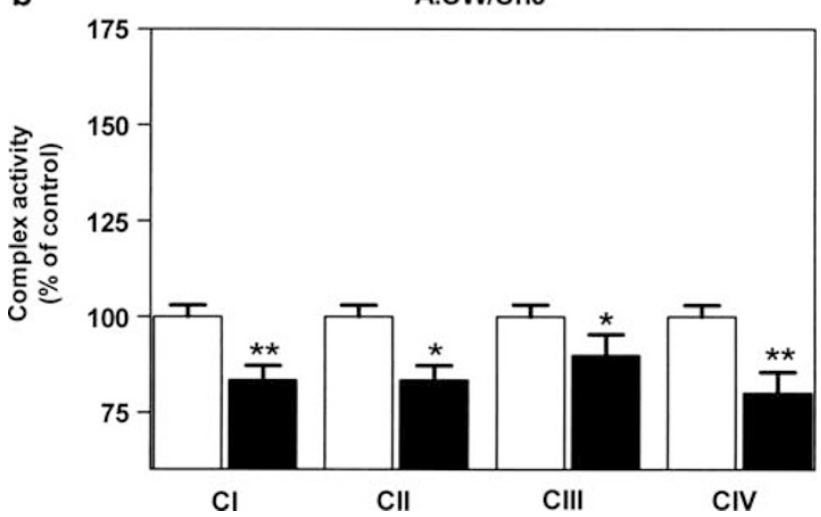

Figure 2 Respiratory chain complex activity of $\mathrm{Cl}, \mathrm{CII}, \mathrm{CIII}$ and CIV were measured in isolated mitochondria from uninfected (control, open bars) and CVB3-infected (filled bars) C57BL/6 (a) and A.SW/SnJ hearts (b). Data are shown as percentages of the uninfected controls. ${ }^{\star} P<0.05 ;{ }^{* *} P<0.01$ vs controls.

protein amounts $(181.8 \pm 8.9 \% ; P<0.001)$ (Figures $4 \mathrm{a}$ and $\mathrm{b}$ ). This was accompanied by a remarkable enrichment in caspase 3 protein. The increase equally affected pro-caspase 3 and its active form (Figure 4a) and was therefore combined as total caspase $3(346 \pm 52.2 \% ; P<0.001)$ (Figure $4 \mathrm{~b})$. In addition, TUNEL staining confirmed the presence of apoptotic cells in infected C57BL/6 heart tissue (Figure 4e).

Compared with infected C57BL/6 hearts, Bax and Bcl-2 protein remained unaffected in the infected A.SW/SnJ mouse hearts despite elevated expression of inactive and active caspase $3(179.6 \pm 5.4 \% ; P<0.01)$ (Figures $4 \mathrm{c}$ and $\mathrm{d})$ which, however, appeared to be lower than in the infected C57BL/6 hearts $(P<0.001)$. No apoptotic nuclei were found in the infected A.SW/SnJ mouse hearts (Figure 4e). Thus, the two strains revealed significant differences in the regulation of mitochondrion-related apoptosis in the acute phase of MC.

\section{Correlation Between Myocardial CVB3 Titer and Mitochondrial Parameters}

We checked for a direct relationship between mitochondrial function and myocardial CVB3 load by correlation analyses including the data from C57BL/6 and A.SW/SnJ mice.
Low myocardial CVB3 titers were closely related to elevated CI $(r=-0.89 ; \quad P<0.001)$ (Figure $5 \mathrm{a})$ and CIII activity $(r=-0.75 ; P<0.001)$ (Figure 5b). In addition, reduced catalase expression $(r=0.87 ; P<0.001)$ (Figure $5 \mathrm{c}$ ) was correlated with a depressed virus load in the infected hearts. Increased caspase 3 expression was further linked to virus reduction $(r=-0.71 ; P<0.01)$ (Figure $5 \mathrm{~d})$.

Thus, an imbalance in RC activities in CVB3-infected hearts that are associated with oxidative stress and apoptosis were linked to a reduced myocardial CVB3 load.

\section{Mitochondrial Parameters in Chronic MC}

We investigated mitochondrial function in the chronic phase of MC 90 days p.i. that was only evident in A.SW/SnJ mice, while the C57BL/6 mice recovered completely. Although immunological infiltration was no longer histologically detectable in infected A.SW/SnJ mouse hearts, the myocardial immune response was still active as seen by an increased level of TGF $\beta$, while TNF $\alpha$ and IL- $1 \beta$ had normalized (Figure 6a). Replicating CVB3 was no longer observed by plaque-forming assay (data not shown).

A.SW/SnJ hearts showed permanent restriction of CI activity, whereas CII, CIII and CIV activity reached normal levels (Figure 6b). SOD2 and catalase expression and LPO levels returned to normal (data not shown). Although procaspase 3 expression (Figure $6 \mathrm{c}$ ) was significantly decreased $(40.2 \pm 7.4 \% ; P<0.01)$ and no active caspase 3 or apoptotic nuclei were detectable (data not shown), both the anti- and proapoptotic markers Bcl-2 $(145.1 \pm 14.7 \% ; P<0.05)$ and Bax $(133.1 \pm 3.9 ; P<0.001)$ were elevated.

Thus, resistant C57BL/6 mice recovered completely with normalization of all intracellular parameters described above. A.SW/SnJ mice disclosed a lasting activation of immune response and disturbance in mitochondrial parameters.

\section{DISCUSSION}

CVB3-resistant C57BL/6 and A.SW/SnJ mice that exhibited a different quality of immune response and ability in virus elimination revealed distinct mitochondrial function within the infected myocardium. C57BL/6 mice, which quickly eliminate CVB3 from the heart and recover from $\mathrm{MC}$, showed a strong imbalance in the myocardial mitochondrial $\mathrm{RC}$, exhaustion of the antioxidative system, pronounced oxidative stress and strong mitochondrion-related apoptosis, which generates an antiviral intracellular environment in the acute phase of MC. C57BL/6 mice showed high levels of $\mathrm{TNF} \alpha$, IL- $1 \beta$ and TGF $\beta$, cytokines that are known to affect the energy metabolism. ${ }^{15}$ Interestingly, myocardial IL- $1 \beta$ and $\operatorname{TGF} \beta$ transcription were much lower in infected A.SW/SnJ mice developing chronic MC. They displayed decreased but balanced RC activities, increased catalase expression as well as suppressed oxidative damage and mitochondrial apoptosis in acute MC.

Cardiac electron transfer over the mitochondrial RC significantly impacts the modulation of apoptosis, ${ }^{8}$ a central 


\section{Table 1 Comparison of RC complex subunit expression of CVB3-infected C57BL/6 vs A.SW/SnJ hearts}

tame- Accession no.

Name

Accession no.

Ratio*

P-value

Complex 1

NADH dehydrogenase [ubiquinone] 1 alpha subcomplex subunit 1 (NDUFA1)

NADH dehydrogenase [ubiquinone] 1 alpha subcomplex subunit 2 (NDUFA2)

$\begin{array}{lll}\text { O35683 } & 1.858 & <0.001 \\ \text { Q9CQ75 } & 1.142 & <0.05 \\ \text { Q9Z1P6 } & 1.357 & <0.001 \\ \text { Q9CQC7 } & 1.153 & <0.001 \\ \text { Q9CQJ8 } & 1.295 & <0.05 \\ \text { Q9DCS9 } & 1.130 & <0.01 \\ \text { O09111 } & 1.228 & <0.01 \\ \text { Q9CXZ1 } & 1.324 & <0.05 \\ \text { Q99LY9 } & 1.233 & <0.001 \\ \text { Q8BK30 } & 1.221 & <0.01 \\ \text { Q59J78 } & 3.238 & <0.001\end{array}$

Complex II

Succinate dehydrogenase cytochrome b560 subunit (SDHC)

Q9CZB0

Complex III

Cytochrome b-c1 complex subunit 5 (UQCRFS1)

$\begin{array}{ccc}\text { Q9CR68 } & -1.135 & <0.05 \\ \text { Q8R1I1 } & -1.385 & <0.001 \\ \text { Q9D0M3 } & -1.227 & <0.05\end{array}$

Cytochrome $\mathrm{c} 1$, heme protein (CYC1)

Q9D0M3

0.05

Complex IV

Cytochrome c oxidase subunit 7A-related protein (COX7A2L)

Cytochrome c oxidase polypeptide 7A1 (COX7A1)

$\begin{array}{lll}\text { Q61387 } & -6.357 & <0.001 \\ \text { P56392 } & -1.153 & <0.05\end{array}$

${ }^{*}$ The ratio was determined by dividing the observed intensities in mitochondrial protein extracts of CVB3-infected C57BL/6 hearts by those of A.SW/SnJ hearts. Minus symbol: amount of subunit is lower in C57BL/6 than in A.SW/SNJ mice.

a

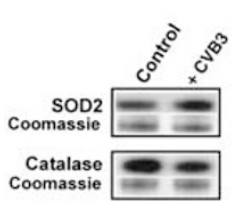

d

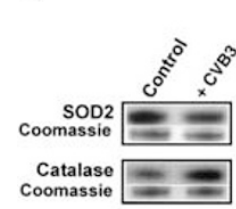

b

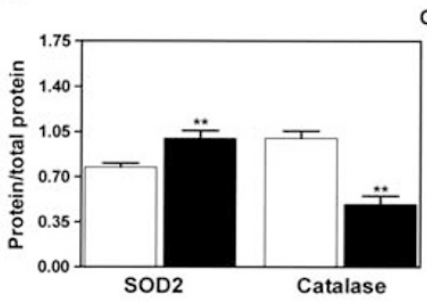

e

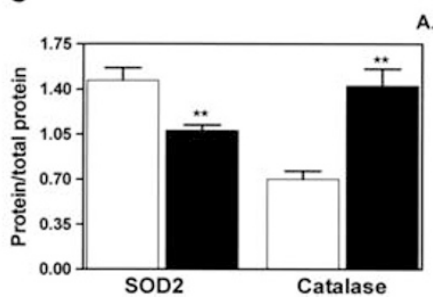

C

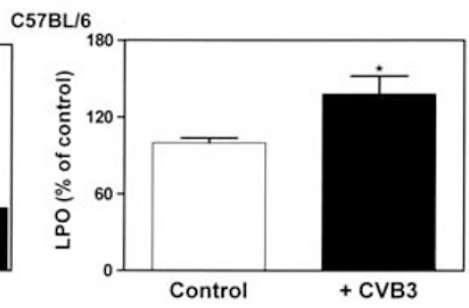

f A.SWISnJ

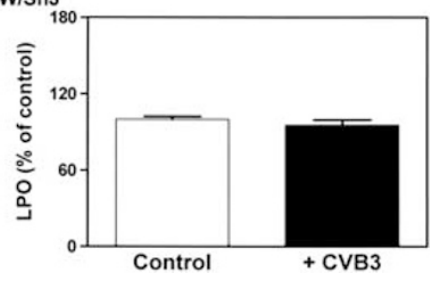

Figure 3 Protein levels of mitochondrial SOD2 and cytosolic catalase were determined in uninfected (control; open bars) and infected (+ CVB3; filled bars) C57BL/6 (a, b) and A.SW/SnJ mice (d, e) by western blotting. (a, d) show representative western blots of SOD2 and catalase (upper panels). Specific signals were normalized against total loaded protein visualized by Coomassie blue staining (lower panels). Myocardial lipid peroxidation was spectroscopically measured in C57BL/6 (c) and A.SW/SnJ (f) hearts. ${ }^{\star} P<0.05 ;{ }^{* *} P<0.01$ vs controls. 
effective host response to minimize damage because of viral infection, while quickly clearing the invading pathogen. Imbalance of RC additionally supports apoptosis by promoting the production of radicals damaging structural and functional macromolecules. ${ }^{6,7}$ Underscoring this concept, our correlation analysis reveals a strong link between unbalanced mitochondrial energy metabolism, a repressed antioxidative system, enhanced mitochondrion-related apoptosis and low myocardial CVB3 titer in the acute phase of MC. Although the two infected mouse strains showed an equal grade of inflammation in acute MC, long-lasting differences in the cytokine patterns clearly pointed to a distinct quality of local immune response. TGF $\beta$, which is well known for having a significant impact on the mitochondrial function ${ }^{16}$ and for exerting a suppressing effect on CVB3 infection, ${ }^{17,18}$ was differently regulated in C57BL/6 and A.SW/SnJ mice. A close association between TGF $\beta$ level and increase in CIII activity, Bax and Bcl-2 as well as a decrease in myocardial CVB3 titer confirms the significance of TGF $\beta$ in CVB3-induced MC. Thus, not only the quantity of infiltrating cells but above all the quality of the responses is associated with the diversity of intracellular processes and virus elimination. Conversely, intracellular processes directly related to the mitochondrial energy metabolism have been known to determine the release of cytokines, thus affecting the immune system and virus infection. ${ }^{19}$

C57BL/6 mice disclosed a highly imbalanced RC in the acute phase of MC characterized by enhanced CI and CIII activity but decreased CII and normal CIV activity. Changes in $\mathrm{RC}$ activity are in part related to altered subunit expression of the RC complexes. The protein amount of several CI subunits was higher in infected C57BL/6 than in A.SW/SnJ hearts. This may not only be due to a downregulation in CI subunits in A.SW/SnJ hearts as seen in infected permissive $\mathrm{A} / \mathrm{J}$ mice $^{20}$ but also to subunit upregulation in $\mathrm{C} 57 \mathrm{BL} / 6$ mice. In this regard, IL- $1 \beta$ has been shown to enhance CI subunit expression in vitro. ${ }^{21,22}$ In agreement with this, our data revealed a significant association between IL- $1 \beta$ mRNA expression and CI activity in the infected hearts. Most of the affected CI subunits are accessory proteins that do not directly belong to CI's catalytic core. ${ }^{23}$ However, mutations in accessory subunits like NDUFA1, NDUFA2, NDUFS4 have been shown to affect CI activity, ${ }^{24}$ clearly indicating their relevance for CI functionality. In contrast to CI, the subunit expression of CIII and CIV acted contrarily to their activity, suggesting a compensatory effect against activity-inhibiting agents or RC complex modifications like oxidation and phosphorylation as shown in inflamed hearts and cytokinetreated isolated cells. ${ }^{25-27}$ Thus, the regulation of the RC complex activities occurred at least in part on the translational level and appears to be related to different myocardial cytokine expression patterns within the infected mouse strains.

The profound imbalance in RC function, including enhanced CI and CIII activity, involves a high risk of elevated oxidative stress in infected C57BL/6 hearts. CI and CIII activities are the main producers of ROS within the mitochondria. ${ }^{7}$ Huang et al. ${ }^{28}$ reported that increased CI and CIII subunit expression leads to ROS generation that could be blocked by CI-specific knockdown. ROS modify mitochondrial proteins and disturb mitochondrial integrity, which finally leads to apoptosis ${ }^{29}$ unless it is sufficiently neutralized by the antioxidative enzyme system. Although the mitochondrial antioxidative enzyme SOD2 that converts $\mathrm{O}_{2}^{-}$ to $\mathrm{H}_{2} \mathrm{O}_{2}$ was moderately enhanced in infected C57BL/6 mice, the expression of catalase that neutralizes $\mathrm{H}_{2} \mathrm{O}_{2}$ was remarkably reduced. Such conditions support ROS accumulation that is associated with oxidative stress and apoptosis as observed in infected C57BL/6 hearts. Similar findings were observed in human MC by Xie et al. ${ }^{30}$ They demonstrated that oxidative stress evidenced by elevated plasma LPO and nitric oxide was associated with a decrease in antioxidative enzymes like SOD. Moreover, catalase was severely aggravated in patients suffering from acute enterovirus-associated MC. Oxidative stress is often associated with mitochondrionrelated apoptosis and has also been observed in infected C57BL/6 mice, characterized by increased Bax, Bcl-2 as well as caspase 3 activation and DNA degradation. Thus, imbalanced RC in resistant mice is linked to a prooxidative and proapoptotic environment, both conditions that support virus elimination.

Interestingly, we observed completely different conditions in infected A.SW/SnJ hearts that were unable to efficiently remove CVB3 from the heart. Permissive A.SW/SnJ mice coordinately downregulated all RC complex activities, which is thought to decrease the flow of electrons over the $\mathrm{RC}^{31}$ and lower the risk of electron misrouting and ROS production. In addition, an activated antioxidative system shown by increased catalase expression blocked the generation of oxidative stress and thus LPO production. High catalase expression has been shown to have cardioprotective effects in other heart diseases such as ischemia. ${ }^{32,33}$ In addition, enhanced glutathione peroxidase and thioredoxin transcription, additional antioxidative stress components, were found in CVB3-infected permissive A/J mice with acute MC. ${ }^{20}$ SOD2 expression was reduced in infected A.SW/SnJ hearts but was still as high as in infected C57BL/6 mice. Thus, A.SW/SnJ mice suppressed oxidative stress by reducing electron flow over the RC and sustain the antioxidative system. Suppression of oxidative stress preserved mitochondrial integrity and aborted severe mitochondrion-related apoptosis indicated by unaffected Bax and Bcl-2 expression in these animals. The increase in myocardial caspase 3 expression and activation in infected A.SW/SnJ mice was much lower than in infected C57BL/6 mice and considered to be induced by the extrinsic mitochondrion-independent pathway. ${ }^{34}$ However, its activation was not sufficient enough to cause DNA degradation. Hence, the suppression of intracellular oxidative stress and intrinsic apoptosis in the early phase of MC is accompanied by high virus replication in the heart. 

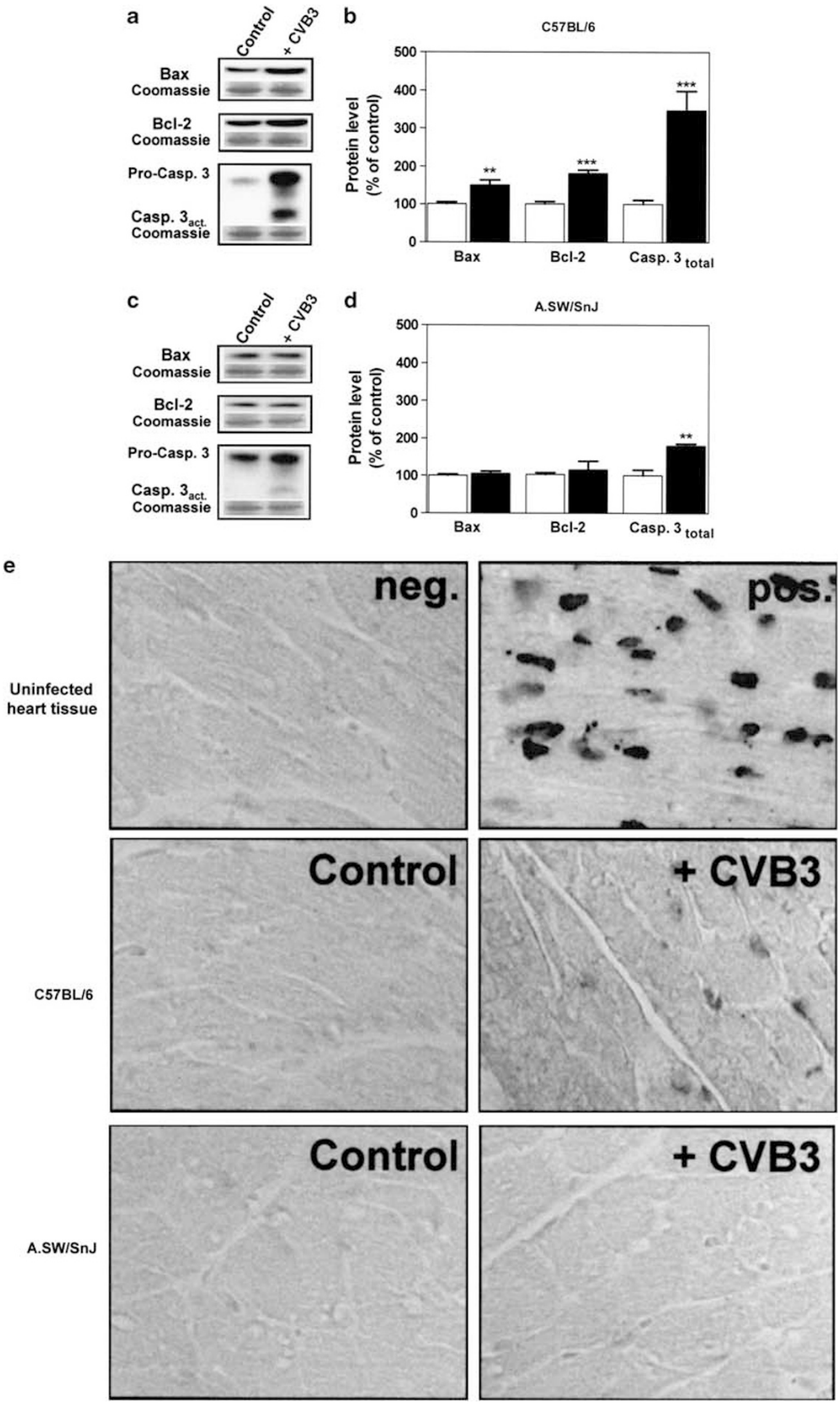
Disturbed energy metabolism has also been observed in chronic experimental and human viral MC. ${ }^{35-37}$ So far it was ambiguous at what point energetic restrictions occur in the progression of chronic MC and whether they are the cause or result of cardiac impairment. Our findings demonstrate that alterations in energy production arise already in the acute phase of MC and persist up to the chronic phase in permissive A.SW/SnJ mice, whereas resistant C57BL/6 mice recovered completely.

In the chronic phase of MC, A.SW/SnJ mice evidenced elevated Bax and Bcl-2 expression, while caspase 3 expression was significantly reduced and no apoptotic nuclei were visible, showing that extensive programmed cell death did not occur at that time point. No replicating virus was detectable within the heart at that time, but the immune response was still active. Schulze et al. ${ }^{36}$ have previously demonstrated a tight correlation between disturbed energy metabolism and cardiac performance in CVB3-infected A.SW/SnJ mice, indicating the significance of mitochondrial function and the pathophysiology of the disease. Thus, an imbalanced energy metabolism significantly affects not only the antiviral process but also the myocardial function contributing to the severity of the disease. Disturbance in energy metabolism has also been observed in human inflammatory and dilated cardiomyopathy, ${ }^{13,35}$ assuming that similar processes occur in humans.
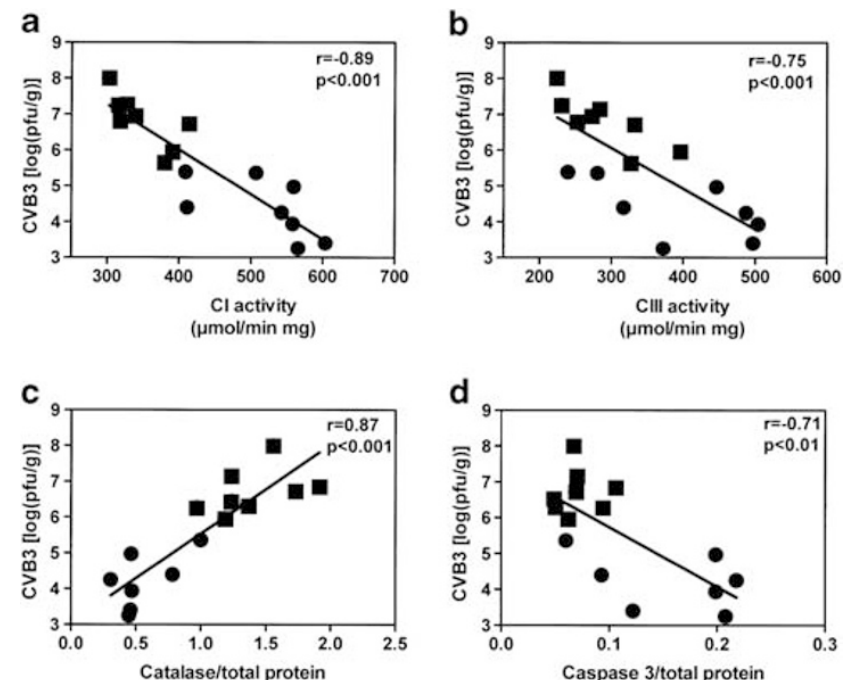

Figure 5 The correlation between myocardial CVB3 load shown as $\log$ (p.f.u./g tissue) and (a) $\mathrm{Cl}$ activity, (b) Clll activity, (c) catalase and (d) caspase 3 expression was determined based on the data received from infected C57BL/6 (black dots) and A.SW/SnJ (black rectangles) mice by linear regression ( $r=$ regression factor, $n=15-16$ ).
Our data may help to explain the findings of Alter and Maisch. ${ }^{38}$ The investigators analyzed myocardial biopsies from patients with MC and DCM. They did not find apoptosis in patients with enteroviral genome persistence. In contrast, they observed elevated concentrations of soluble Fas-receptors repressing the extrinsic pathway of apoptosis only in patients with enterovirus persistence. ${ }^{39}$ Thus, inhibition of Fas-ligand-induced extrinsic apoptosis blocked by soluble Fas-receptors can impair virus clearance and prolong
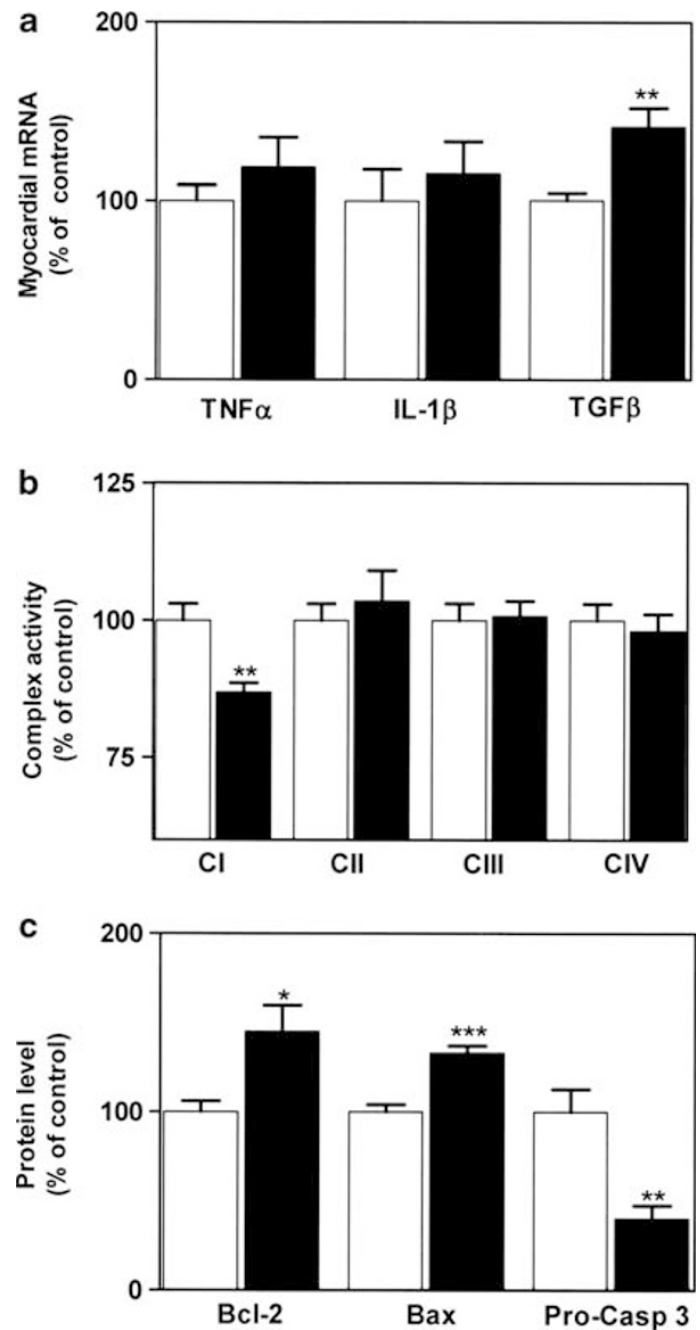

Figure 6 Biochemical abnormalities in the myocardial cytokine pattern (a), the respiratory chain complex activity (b) and expression of Bcl-2, Bax, and caspase-3 (c) were found in infected A.SW/SnJ mice (filled bars) in comparison with uninfected controls (open bars) 90 days p.i. All parameters returned to baseline in infected C57BL/6 mice and were not graphically presented. Data are shown as percentages of the uninfected controls. ${ }^{\star} P<0.05 ;{ }^{*} P<0.01 ;{ }^{* *} P<0.001$ vs controls.

Figure 4 Expression of pro- and antiapoptotic markers was determined in uninfected (control; open bars) and infected (+CVB3; filled bars) C57BL/6 $(\mathbf{a}, \mathbf{b})$ and A.SW/SnJ mice (c, d) by western blotting. (a, c) show representative western blot analyses of Bax, Bcl-2 as well as pro-caspase 3 and active caspase 3 (upper panels). Specific signals were normalized against total loaded protein visualized by Coomassie blue staining (lower panels). Graph (b, d) display the quantification of proteins in \% of uninfected controls. (e) Detection of TUNEL-positive cardiomyocytes in uninfected (control) and CVB3-infected ( + CVB3) C57BL/6 and A.SW/SnJ hearts. Negative control of the TUNEL assay was confirmed by staining the heart tissue section without primary antibody. Positive control of the TUNEL assay was performed by treating the heart sections with DNase before staining. ${ }^{* * P}<0.01 ;{ }^{* *} P<0.001$ vs controls. 
viral replication, leading to a potentially poorer outcome of the disease. Consistent with these observations, Abbate et al. ${ }^{40}$ recently observed that a high rate of cardiomyocyte apoptosis supports the functional recovery of MC patients by assuming an efficient elimination of infected and damaged cells.

Thus, mitochondrial processes most probably regulated by immunological factors determine about virus clearance or persistence. We conclude that coordinated analyses of virus presence and immunological parameters in combination with mitochondrial parameters describing energy metabolism, oxidative stress and apoptosis may allow a better prognosis of the disease course in patients with MC. The data also determine mitochondria as potential targets for therapeutic intervention in CVB3-induced MC.

\section{ACKNOWLEDGEMENTS}

This study was supported by the 'Sonderforschungsbereich SFB, Transregio 19, Deutsche Forschungsgemeinschaft (DFG)'. L Ebermann was supported by a PhD scholarship from Freie Universität Berlin, Berlin, Germany. H Zeichhardt gratefully acknowledges partial support by the 'Otto-Kuhn-Stiftung im Stifterverband für die Deutsche Wissenschaft'.

\section{DISCLOSURE/CONFLICT OF INTEREST}

The authors declare no conflict of interest.

1. Knowlton KU. CVB infection and mechanisms of viral cardiomyopathy. Curr Top Microbiol Immunol 2008;323:315-335.

2. Kuhl $U$, Pauschinger $M$, Noutsias $M$, et al. High prevalence of viral genomes and multiple viral infections in the myocardium of adults with 'idiopathic' left ventricular dysfunction. Circulation 2005;111:887-893.

3. Arnoult D, Carneiro L, Tattoli I, et al. The role of mitochondria in cellular defense against microbial infection. Semin Immunol 2009;21:223-232.

4. Benedict CA, Norris PS, Ware CF. To kill or be killed: viral evasion of apoptosis. Nat Immunol 2002;3:1013-1018.

5. Boya P, Roumier T, Andreau K, et al. Mitochondrion-targeted apoptosis regulators of viral origin. Biochem Biophys Res Commun 2003;304:575-581.

6. Chen Q, Vazquez EJ, Moghaddas S, et al. Production of reactive oxygen species by mitochondria: central role of complex III. J Biol Chem 2003;278:36027-36031.

7. Grivennikova VG, Vinogradov AD. Generation of superoxide by the mitochondrial complex I. Biochim Biophys Acta 2006;1757:553-561.

8. Gustafsson $A B$, Gottlieb RA. Heart mitochondria: gates of life and death. Cardiovasc Res 2008;77:334-343.

9. Brenner D, Mak TW. Mitochondrial cell death effectors. Curr Opin Cell Biol 2009:21:871-877.

10. Leipner C, Grun K, Schneider I, et al. Coxsackievirus B3-induced myocarditis: differences in the immune response of C57BL/6 and Balb/ c mice. Med Microbiol Immunol 2004;193:141-147.

11. Wolfgram LJ, Beisel KW, Herskowitz A, et al. Variations in the susceptibility to Coxsackievirus B3-induced myocarditis among different strains of mice. J Immunol 1986;136:1846-1852.

12. Dorner A, Grunert HP, Lindig V, et al. Treatment of coxsackievirus-B3infected BALB/c mice with the soluble coxsackie adenovirus receptor CAR4/7 aggravates cardiac injury. J Mol Med 2006;84:842-851.

13. Jarreta D, Orus J, Barrientos $A$, et al. Mitochondrial function in heart muscle from patients with idiopathic dilated cardiomyopathy. Cardiovasc Res 2000;45:860-865.

14. Greinacher A, Wesche J, Hammer E, et al. Characterization of the human neutrophil alloantigen-3a. Nat Med 2010;16:45-48.

15. Tatsumi T, Matoba S, Kawahara A, et al. Cytokine-induced nitric oxide production inhibits mitochondrial energy production and impairs contractile function in rat cardiac myocytes. J Am Coll Cardio 2000;35:1338-1346

16. Sanchez-Capelo A. Dual role for TGF-beta1 in apoptosis. Cytokine Growth Factor Rev 2005;16:15-34.
17. Horwitz MS, Knudsen M, llic A, et al. Transforming growth factor-beta inhibits coxsackievirus-mediated autoimmune myocarditis. Viral Immunol 2006;19:722-733.

18. Shi Y, Fukuoka M, Li G, et al. Regulatory $T$ cells protect mice against coxsackievirus-induced myocarditis through the transforming growth factor beta-coxsackie-adenovirus receptor pathway. Circulation 2010;121:2624-2634.

19. Koshiba T, Yasukawa K, Yanagi Y, et al. Mitochondrial membrane potential is required for MAVS-mediated antiviral signaling. Sci Signal 2011;4:ra7.

20. Taylor LA, Carthy CM, Yang D, et al. Host gene regulation during coxsackievirus B3 infection in mice: assessment by microarrays. Circ Res 2000;87:328-334.

21. Wegrzyn $P$, Jura J, Kupiec $T$, et al. A search for genes modulated by interleukin- 6 alone or with interleukin-1beta in HepG2 cells using differential display analysis. Biochim Biophys Acta 2006;1762:319-328.

22. Wegrzyn P, Yarwood SJ, Fiegler N, et al. Mimitin - a novel cytokine-regulated mitochondrial protein. BMC Cell Biol 2009;10:23.

23. Remacle C, Barbieri MR, Cardol P, et al. Eukaryotic complex I: functional diversity and experimental systems to unravel the assembly process. Mol Genet Genomics 2008;280:93-110.

24. Fosslien E. Review: mitochondrial medicine-cardiomyopathy caused by defective oxidative phosphorylation. Ann Clin Lab Sci 2003;33:371-395.

25. Huttemann M, Lee I, Samavati L, et al. Regulation of mitochondrial oxidative phosphorylation through cell signaling. Biochim Biophys Acta 2007:1773:1701-1720

26. Samavati L, Lee I, Mathes I, et al. Tumor necrosis factor alpha inhibits oxidative phosphorylation through tyrosine phosphorylation at subunit I of cytochrome c oxidase. J Biol Chem 2008;283:21134-21144.

27. Zell R, Geck P, Werdan K, et al. TNF-alpha and IL-1 alpha inhibit both pyruvate dehydrogenase activity and mitochondrial function in cardiomyocytes: evidence for primary impairment of mitochondrial function. Mol Cell Biochem 1997;177:61-67.

28. Huang $G$, Chen $Y$, Lu $H$, et al. Coupling mitochondrial respiratory chain to cell death: an essential role of mitochondrial complex I in the interferon-beta and retinoic acid-induced cancer cell death. Cell Death Differ 2007;14:327-337.

29. Circu ML, Aw TY. Reactive oxygen species, cellular redox systems, and apoptosis. Free Radic Biol Med 2010;48:749-762.

30. Xie B, Zhou JF, Lu Q, et al. Oxidative stress in patients with acute coxsackie virus myocarditis. Biomed Environ Sci 2002;15:48-57.

31. Korshunov SS, Skulachev VP, Starkov AA. High protonic potential actuates a mechanism of production of reactive oxygen species in mitochondria. FEBS Lett 1997;416:15-18.

32. Burwell LS, Nadtochiy SM, Brookes PS. Cardioprotection by metabolic shut-down and gradual wake-up. J Mol Cell Cardiol 2009;46:804-810.

33. Woo YJ, Zhang JC, Vijayasarathy $C$, et al. Recombinant adenovirusmediated cardiac gene transfer of superoxide dismutase and catalase attenuates postischemic contractile dysfunction. Circulation 1998;98 (19 Suppl):II255-II260.

34. Movassagh M, Foo RS. Simplified apoptotic cascades. Heart Fail Rev 2008:13:111-119.

35. Dorner $\mathrm{A}$, Pauschinger $\mathrm{M}, \mathrm{Schwimmbeck} \mathrm{PL}$, et al. The shift in the myocardial adenine nucleotide translocator isoform expression pattern is associated with an enteroviral infection in the absence of an active T-cell dependent immune response in human inflammatory heart disease. J Am Coll Cardiol 2000;35:1778-1784.

36. Schulze K, Witzenbichler B, Christmann C, et al. Disturbance of myocardial energy metabolism in experimental virus myocarditis by antibodies against the adenine nucleotide translocator. Cardiovasc Res 1999;44:91-100.

37. $\mathrm{Xu} \mathrm{J}$, Nie HG, Zhang XD, et al. Down-regulated energy metabolism genes associated with mitochondria oxidative phosphorylation and fatty acid metabolism in viral cardiomyopathy mouse heart. Mol Biol Rep 2011;38:4007-4013.

38. Alter P, Maisch B. Escape from cardiomyocyte apoptosis by enterovirus persistence due to elevated soluble Fas-receptors. Z Kardiol 2004;93:524-532.

39. Alter $\mathrm{P}$, Jobmann $\mathrm{M}$, Meyer $\mathrm{E}$, et al. Apoptosis in myocarditis and dilated cardiomyopathy: does enterovirus genome persistence protect from apoptosis? An endomyocardial biopsy study. Cardiovasc Pathol 2001;10:229-234.

40. Abbate A, Sinagra G, Bussani R, et al. Apoptosis in patients with acute myocarditis. Am J Cardiol 2009;104:995-1000. 